\title{
Théories des relations publiques et théories de la communication
}

\section{Axel Gryspeerdt}

\section{(2) OpenEdition}

12 Journals

Édition électronique

URL : http://journals.openedition.org/communicationorganisation/1647

DOI : 10.4000/communicationorganisation. 1647

ISSN : $1775-3546$

Éditeur

Presses universitaires de Bordeaux

\section{Édition imprimée}

Date de publication : 1 novembre 1993

ISSN : 1168-5549

\section{Référence électronique}

Axel Gryspeerdt, "Théories des relations publiques et théories de la communication », Communication et organisation [En ligne], 4 | 1993, mis en ligne le 26 mars 2012, consulté le 21 septembre 2020. URL: http://journals.openedition.org/communicationorganisation/1647; DOI : https://doi.org/10.4000/ communicationorganisation. 1647

Ce document a été généré automatiquement le 21 septembre 2020.

(c) Presses universitaires de Bordeaux 


\title{
Théories des relations publiques et théories de la communication
}

\author{
Axel Gryspeerdt
}

1 Contrairement à ce que l'on pense communément, les relations publiques et les théories de la communication n'ont pas toujours fait bon ménage. On a même l'impression qu'elles ont vécu de manière séparée pendant de longues années. L'Europe surtout a paru privilégier la voie de la ségrégation plutôt que celle de l'enrichissement et de la fécondation mutuelles.

2 Même là où elles coexistent dans une même institution d'enseignement et de recherche, communication et relations publiques sont d'ailleurs souvent gérées de manière distincte avec offre de formations spécifiques et publications spécialisées.

3 Certains groupes extérieurs à la pratique professionnelle s'interrogent actuellement sur cette distance et sur les travers qu'elle risque d'instaurer. Destinataires des actions de relations publiques, étudiants ou futurs professionnels se demandent ainsi en quoi le secteur de la communication d'entreprise serait tellement spécifique que ni les théories de l'interaction ni celles de la communication ne pourraient l'éclairer ou l'enrichir, à l'instar de ce qui se fait par exemple dans le domaine de la communication politique?

4 Il faut bien constater qu'au cours des dernières années, le champ professionnel, mais aussi académique, de réflexion sur les relations publiques a eu plus vite tendance à s'insérer au sein des pratiques et des théories du management que parmi celles de la communication et des sciences sociales. Ce qui peut d'ailleurs expliquer pourquoi les relations publiques ont eu souvent du mal à être considérées comme une discipline des sciences sociales et ce qui n'a guère facilité les relations entre les communicateurs d'entreprise et les chercheurs en sciences sociales.

Le présent dossier offre une opérationnalisation de ce possible. Que se passerait-il si les chercheurs en sciences sociales et les chercheurs en communication se hasardaient à confronter leurs approches théoriques aux pratiques de communication dans les organisations? Qu'en serait-il de la confrontation entre les théories et les opérations concrètes? Et de l'interrogation des situations de relations publiques par les théories existantes ou par certains concepts empruntés aux sciences humaines? Pourquoi ne 
pas concevoir dès le départ l'entreprise dans les liens - l'ensemble des liens - qu'elle entretient avec la totalité du système social et avec les pratiques de la vie en société ?

En quelque sorte le dossier est un pari. Bien sûr, il n'est pas purement isolé. Sa démarche présente notamment des affinités avec d'autres dossiers de la revue Communication \& Organisation, toujours soucieuse de faire la jonction entre les pratiques et les théories, entre les professionnels et les chercheurs.

7 Elle en présente aussi avec d'autres publications rédigées par des groupes de recherche que l'on doit bien appeler minoritaires, puisque la voie dominante se situe ailleurs.

8 Mais simultanément, par son ambition propre, il se présente comme un premier jalon. En réunissant des articles qui proposent des pistes d'investigation, des voies parfois nouvelles, voire des aventures intellectuelles. Certains articles du dossier ne donnent guère des résultats de recherches élaborées. Leur seule volonté est d'ouvrir quelques perspectives, de lancer, ce que Yves Winkin, dans le texte qu'il propose ici, appelle des stratégies de ruptures.

Même si les auteurs, dont les textes sont réunis dans le dossier, ont travaillé dans des temps et dans des lieux institutionnels différents, leurs préoccupations se rejoignent. Chacun à sa manière propose des perspectives de recherche, qu'on souhaite fécondes pour la connaissance des phénomènes de communication des organisations. Ils essayent de nouveaux modèles, que certains d'entre-eux importent d'autres domaines que ceux des relations publiques traditionnelles ou modernes.

10 Ainsi l'histoire, la sociologie, la théorie des systèmes, l'ethnosociologie, la science du désordre, pour ne citer que quelques-unes des approches retenues, fournissent-elles, quelques-unes des pistes proposées par les auteurs pour confronter les pratiques ou réinterroger les relations publiques. La question est moins de voir si ces dernières résisteront à ces confrontations, que de mieux comprendre les phénomènes de communication d'entreprise, dans leur complexité actuelle, à l'aide de ces diverses interrogations.

11 Mais il s'agit dans le même temps de voies "dérangeantes " puisqu'elles quittent délibérément la perspective auto-légitimée et auto-reproductrice des tenants d'un discours propre aux relations publiques et au management, pour proposer de nouvelles aventures.

Deux principaux lieux, ou deux rencontres de chercheurs et de professionnels intéressés par des enjeux théoriques ont suscité l'idée du dossier.

13 1. En mai 1992, eut lieu, à Louvain-la-Neuve, une rencontre de chercheurs en communication de diverses universités, réunis dans la perspective de confronter les théories des relations publiques et les théories de l'interaction et de la communication. Patronnée par le Fonds National belge de la Recherche Scientifique (FNRS), elle voulait « envisager les relations publiques à la fois comme des représentations sociales, des pratiques discursives et des séquences de comportements interactionnels, qu'il faut analyser à la lumière des théories systémiques et des théories interactionnistes qui irriguent aujourd'hui les théories de la communication ».

Depuis lors, une quinzaine de chercheurs belges interpellés par la communication d'entreprise se sont promis de se réunir annuellement pour échanger leurs réflexions et confronter leurs analyses sur les relations publiques, sous l'égide du Groupe de contact du FNRS intitulé "Théories des relations publiques et théories de la communication », animé par Alain Nivarlet et Yves Winkin (Université de Liège) et par 
moi-même (U.C.L.). La première rencontre a principalement porté sur les phénomènes de représentation et d'admiration. La seconde sur les questions d'apparence et de fonds dans les relations publiques.

2. Antérieurement à cette réflexion commune de 1992, un colloque avait réuni quelques dizaines de chercheurs et de professionnels de la communication d'organisation. Pendant deux jours de séances plénières et de carrefours, ils avaient traité de questions relatives aux manières modernes d'exprimer l'entreprise. Plusieurs publications se sont faites dans la foulée de ce colloque, qui rendait hommage à un des premiers enseignants belges des relations publiques, le Professeur Vincent Levaux, admis à l'éméritat.

Ces questions d'expression d'entreprise, ou, si l'on préfère, le "dire de l'entreprise ", ont fait notamment l'objet d'un dossier, paru dans le $n^{\circ} 3$, tome XXIX, mars 1990, de la revue Reflets et perspectives de la vie économique, consacré au colloque de Louvain-laNeuve intitulé "Dire l'entreprise" et publié à Bruxelles. Ce dernier contient des articles de Frédéric Antoine, Daniel Bodson, Vincent Defourny, Thierry De Smedt, Jules Gritti, Axel Gryspeerdt, Vincent Levaux, Philippe Marion et Yves Winkin.

Néanmoins, certains textes rédigés dans la foulée du colloque, et parfois bien après lui, sont restés inédits, la plupart d'entre-eux consistant en des textes plus théoriques ou plus novateurs, interrogeant les modèles de communication et de développement des organisations.

Ce sont ces textes issus des deux initiatives distinctes, que l'on a souhaité regrouper ici.

Bien qu'ils portent sur des concepts et des approches différentes, ils s'inscrivent chacun dans la perspective «d'alternatives à une vision utilitariste et problématique de la communication » qui a longtemps dominé.

La question principale qu'ils abordent dans leurs écrits, le point de départ de leur analyse n'est pas tellement de chercher à voir comment atteindre une bonne image de firme, comment lui assurer une bonne visibilité, ou comment augmenter sa renommée et celle de ses dirigeants, voire de ses idées et de ses valeurs, toutes questions relevant $\mathrm{du}$ «comment» et du lien entre la communication et le management, mais de s'interroger sur l'existence d'un domaine d'étude appelle "relations publiques» constitué - ou constituable - en champ scientifique et d'en percevoir les origines, les modalité et les évolutions.

21 Jusqu'à présent, le thème principal de la littérature consacrée aux relations publiques a sans doute trop tourné autour de la préoccupation professionnelle de départ, qui est liée à la maxime des premiers praticiens : «comment bien faire et comment le faire savoir "

22 En même temps qu'elle a tenté d'y apporter des réponses, variables selon les périodes et les lieux, cette littérature a valorisé et conforté les démarches professionnelles. Elle est, en outre, intervenue dans le système de représentations et de croyances collectives. Et ceci avec autant plus de poids que cette approche s'est donnée à percevoir comme dominante, sinon exclusive.

23 N'y avait-t-il pas là un phénomène étonnant - et détonnant -, qu'il convenait de dénoncer? Phénomène propre au secteur des relations publiques, car dans d'autres domaines des sciences humaines, on trouve une démarche analytique davantage préoccupée de modéliser leur fonctionnement, de les lier à l'histoire des idées (même 
en management et en marketing), et de permettre la présentation de "discours multiples et contradictoires ".

Ainsi, des pistes alternatives de recherche s'avèrent-elles nécessaires à creuser si on veut que les relations publiques soient fertilisées par les sciences humaines et y puisent une - ou plusieurs - méthodologie d'approche au bénéfice de tous.

Quand elles ambitionnent par exemple d'étudier les relations publiques en termes de rites, de rituels d'interaction et de créations de champs scientifiques, les pistes de recherches ouvertes par les chercheurs du dossier vont effectivement dans ce sens.

Basées sur un « questionnement » des relations publiques, les approches alternatives au modèle purement managérial permettent notamment d'inclure dans le domaine de la communication d'entreprise des pratiques habituellement non-recensées car sans guère d'existence ou de fondement professionnel légitimé, mais néanmoins largement appliquées car répétitives.

27 Ainsi en est-il, par exemple, du vernissage d'expositions ou de certains éléments de communication dite interne des entreprises. Il en a été aussi de même pendant longtemps de certaines formes d'accueil ou de protocole, actuellement plus construites et professionnellement soignées.

28 Il s'agit là de thèmes qui relèvent de la communication d'organisation tout en étant moins couverts par les spécialistes des relations publiques que les conférences de presse, ou que la confection de messages élaborés. Sans doute n'y a-t-il pas lieu de les ignorer, mais en plus, il convient de leur consacrer des efforts de recherche susceptibles de contribuer à une meilleure connaissance des opérations de relations publiques. Même si ces thèmes présentent, a priori moins de visibilité que d'autres thèmes.

29 Il s'agit en quelque sorte de se démarquer de la règle implicite selon laquelle seules les activités menées par des professionnels ayant fonction de communication ou celles entreprises par des agences extérieures de communication sont prises en compte dans les modèles d'analyse et de fonctionnement.

30 Ce n'est d'ailleurs pas une raison pour ignorer les thèmes les plus fréquents de la communication d'entreprise. Il s'agit de poser de nouvelles questions sur eux aussi et de démonter les relations qui s'instaurent entre les divers acteurs concernés.

Les textes de Gérard Derèze, d'Alain Nivarlet et d'Yves Winkin s'inscrivent nettement dans cette perspective. Nathalie Heinich quant à elle ouvre une réflexion de type plus théorique sur la nature des relations publiques.

Il est bon de se rappeler que les relations publiques ne sont pas nées de rien et qu'elles ne peuvent avoir la prétention d'avoir tout inventé. Il y a lieu, dès lors, de rechercher et d'insister sur des connexions à faire avec d'autres représentations, d'autres concepts et d'autres pratiques et à rechercher les points de jonction avec la société civile ou domestique. Ceci aussi les modèles d'analyse de la communication politique l'ont intégré depuis longtemps.

Enfin, il s'agit surtout de pouvoir rendre publique une réflexion dont les bénéficiaires ne soient pas uniquement les milieux professionnels. Mais qui puisse être utile à tous ceux qui sont concernés par les relations publiques, professionnels et consultants, patrons souhaitant avoir des visées à moyen et long termes, étudiants, hommes politiques, décideurs de toutes sortes, enseignants et consommateurs de relations 
publiques... Car dans notre société occidentale, toute personne est concernée par les relations publiques. Toute personne y participe.

34 La communication d'organisation et le management sont trop importants pour être laissés aux seuls professionnels et aux managers, ou même à quelques experts ou conseillers hyperspécialisés de la communication d'entreprise. Certes, ils ne peuvent pas être étudiés sans prendre en compte ces derniers, car leur rôle est prépondérant dans les systèmes de communication mis en place. Ce rôle est même prioritaire. Mais ils doivent, en même temps, être étudiés avec distance et recul.

À leur manière, Pierre de Saint-Georges, Alain Desauvage et Michel Gheude montrent cette communication d'entreprise qui pourrait se faire autre.

Quant à Jean-Louis Lemoigne, Vincent Defourny, Johan De Rycker et François Heinderyckx, ils explorent de nouvelles voies de modélisation qui s'inscrivent dans l'optique de gestion de la complexité des opérations de communication.

37 Confrontation à d'autres théories, importations prudentes de concepts venus des sciences humaines, recherche de compréhension de la complexité, stratégies de ruptures. C'est dans ce cadre que la recherche plus théorique travaillée par des chercheurs qui constituent un groupe encore minoritaire prend, semble-t-il, tout son intérêt. 